\title{
Editorial
}

\section{Designing a molecular analysis of clonality in tumours}

\author{
Salvador J. Diaz-Cano \\ Department of Histopathology and Morbid Anatomy, St Bartholomew's and the Royal London School of Medicine and Dentistry, Whitechapel, London \\ $E I \mid B B, U K$
}

\begin{abstract}
Clonality analysis is used to test malignant transformation and tumour progression. $\mathrm{X}$-chromosome linked clonality assays have been employed for this purpose, but are subject to certain technical limitations. This paper reviews the issues involved and the controls that are necessary to ensure valid interpretation of such analyses.
\end{abstract}

Keywords: clonality; X-chromosome; lyonization; neoplasia

Clonality is an essential attribute of neoplasms and its analysis has been used to test malignant transformation and tumour progression [1,2]. Concordant patterns of genetic markers (X-linked or not) in different tumours suggest that a common progenitor contributed to those lesions and favour, therefore, a multifocal rather than a multicentric origin. These shared genetic alterations also suggest a common cellular origin for biphasic neoplasms [3]. Saxena et al. recently reported a monoclonal pattern in smooth muscle cells and blood vessels of sporadic angiomyolipoma, while the adipose tissue revealed a polyclonal pattern [4]. Based on these findings the authors concluded that the polyclonal adipose tissue is probably metaplastic or reactive. This represents a good example of the application of clonality in tumour biology.

However, some biological and technical issues arise from this article. X-linked clonality assays are based on DNA polymorphism and random X-chromosome inactivation (XCI) in females. Those features enable us to distinguish the maternally from the paternally inherited X-chromosomes [1,5,6]. The mechanisms leading to XCI have not been fully characterized, but DNA methylation might maintain the inactive state, once it is established during early embryogenesis. These methylation patterns are then transmitted by clonal inheritance through the strong preference of mammalian DNA (cytosine-5)-methyltransferase for hemimethylated DNA, involving the promoter regions of alleles on the inactive X-chromosome only [7]. Since $\mathrm{XCI}$ analysis is based on differential DNA methylation of one allele from X-chromosome genes (e.g. human androgen receptor gene), suboptimal enzymatic digestion and abnormal methylation can result in changes of clonality patterns.

According to Lyon's hypothesis, all but one Xchromosomes in a cell are randomly inactivated during early embryogenesis, when the primordial cell pool may comprise as few as 16-30 cells [8]. Given that small number of embryo-destined cells, it reasonable to expect unequal numbers of paternally- and maternallyinherited inactive $\mathrm{X}$-chromosomes, although the $\mathrm{X}$ chromosome is randomly inactivated in each cell. The average Lyonization ratio is close to 50:50 in large cell populations, although individual variation has been found [8]. Skewing towards one allele to an extent that meets the criteria for clonal derivation is consistent with early XCI during embryogenesis (Figure 1).

This finding leads us to consider the selection of appropriate controls to assess the Lyonization ratio in each female. This ratio can also vary from tissue to tissue in the same individual, due to unequal splitting of the cells derived from the primordial cell pool, or to different methylation patterns in different tissues [6,9]. Controls for unequal Lyonization should thus ideally be the most closely related tissue thought not to be involved in the disease process. An essential require-

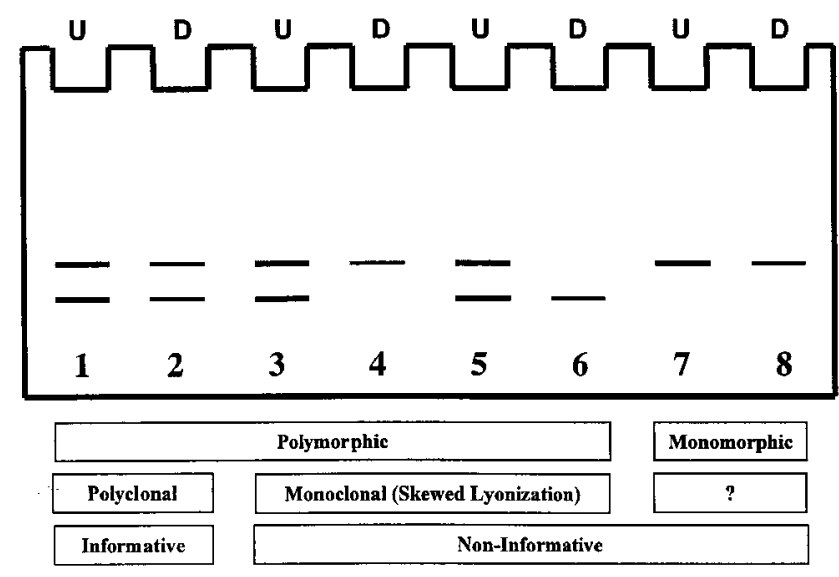

Figure I. Methylation pattern of androgen receptor alleles in control samples. Only polymorphic and polyclonal controls (two allele bands in both undigested and digested samples) are considered informative for clonality assays (lanes I and 2). The remaining possibilities (lanes 3-8) should be excluded from clonality analyses, due to either monoclonal origin of controls (lanes 3-6) or absence of locus polymorphism (lanes 7and 8). $\mathrm{U}=$ undigested sample: $\mathrm{D}=$ digested sample 
ment for clonality analysis is the identification of a polymorphic locus in the normal control (Figure 1). In every case, the tumour sample must be compared with matched controls from the same patient to test the heterozygosity for the marker. Additionally, the individual variability and tissue-related Lyonization ratio require samples of close embryological origin. This feature must be maintained in the digested sample in those tests based on XCI (Figure 1).

Positive allelic imbalances are determined case-bycase, using the skewed data normalized by the allele ratio in matched controls [1,6]. Allelic imbalance analysis is based on the allele ratio and requires densitometric analysis of both allele bands. Therefore, the allele ratio in the target DNA must be maintained in the amplification product, which has to avoid the PCR plateau phase. At this level, any PCR amplification bias should be considered, especially DNA degradation of the larger allele in formalin-fixed, paraffin-embedded tissues and defective amplification of repetitive CG-rich sequences [10-12].

Early XCI occurs randomly and results in a chessboard pattern of cells descended from a common progenitor, which may grow together like a clone (patch size mosaicism). This pattern represents an example of tissue heterogeneity that can also be present in tumours. Sample size is a limiting factor; the lower the cell number, the higher the probability of monoclonal patterns based on patch size mosaicism. This concept becomes particularly important in mixed tumours, where multiple microdissected samples from different tumour areas ( $\geq 100$ cells) and from controls are required to address the question.

Monoclonal patterns support a neoplastic rather than a reactive or hyperplastic process, but are not diagnostic of it. Host cell contamination of tumour samples could give false heterozygous results that would require careful microdissection and microscopic control of the sample collection. However, the pitfalls mentioned above should be always excluded.

Some of these considerations do not appear to have been addressed in the paper of Saxena et al. [4], especially those concerning tests for digestion completion with restriction endonuclease; controls regarding both tumour heterogeneity and their methylation patterns; PCR bias in the amplification of both alleles; tumour heterogeneity and patch size mosaicism; and the meaning of monoclonal and polyclonal patterns.

\section{References}

1. Diaz-Cano SJ, Blanes A, Wolfe HJ. PCR-based techniques for clonality analysis of neoplastic progression. Bases for its appropriate application in paraffin-embedded tissues. Diagn Mol Pathol (in press).

2. Diaz-Cano SJ. Clonality studies in the analysis of adrenal medullary proliferations: application principles and limitations. Endocr Pathol 1998; 9: 301-316.

3. Zhuang Z, Lininger RA, Man YG, Albuquerque A, Merino MJ, Tavassoli FA. Identical clonality of both components of mammary carcinosarcoma with differential loss of heterozygosity. Mod Pathol 1997; 10: 354-362.

4. Saxena A, Alport EC, Custead S, Skinnider LF. Molecular analysis of clonality of sporadic angiomyolipoma. J Pathol 1999; 189: 79-84.

5. Sleddens HF, Oostra BA, Brinkmann AO, Trapman J. Trinucleotide repeat polymorphism in the androgen receptor gene (AR). Nucleic Acids Res 1992; 20: 1427.

6. Mutter GL, Boynton KA. X chromosome inactivation in the normal female genital tract: implications for identification of neoplasia. Cancer Res 1995; 55: 5080-5084.

7. Lyon MF. Some milestones in the history of X-chromosome inactivation. Annu Rev Genet 1992; 26: 16-28.

8. Fialkow PJ. Primordial cell pool size and lineage relationships of five human cell types. Ann Hum Genet 1973; 37: 39-48.

9. Kappler JW. The 5-methylcytosine content of DNA: tissue specificity. J Cell Physiol 1971; 78: 33-36.

10. Mutter GL, Boynton KA. PCR bias in amplification of androgen receptor alleles, a trinucleotide repeat marker used in clonality studies. Nucleic Acids Res 1995; 23: 1411-1418.

11. Diaz-Cano SJ, Brady SP. DNA extraction from formalin-fixed, paraffin-embedded tissues: protein digestion as a limiting step for retrieval of high-quality DNA. Diagn Mol Pathol 1997; 6: 342-346.

12. Diaz-Cano SJ, de Miguel M, Blanes A, Tashjian R, Galera H, Wolfe HJ. Clonality as expression of distinctive cell kinetics patterns in nodular hyperplasias and adenomas of the adrenal cortex. Am J Pathol 2000; 156: 311-319. 